\title{
New-Onset Diabetes Mellitus in Kidney Transplant Recipients Discharged on Steroid-Free Immunosuppression
}

\author{
Fu L. Luan,,$^{1,3}$ Diane E. Steffick, ${ }^{2}$ and Akinlolu O. Ojo ${ }^{1,2}$
}

\begin{abstract}
Background. New-onset diabetes after transplant (NODAT) is a serious complication after kidney transplantation. We studied the relationship between steroid-free maintenance regimens and NODAT in a national cohort of adult kidney transplant patients.

Methods. A total of 25,837 previously nondiabetic kidney transplant patients, engrafted between January 1, 2004, and December 31, 2006, were included in the study. Logistic regression analysis was used to compare the risk of developing NODAT within 3 years after transplant for patients discharged with and without steroid-containing maintenance immunosuppression regimens. The effect of transplant program-level practice regarding steroid-free regimens on the risk of NODAT was studied as well.

Results. The cumulative incidence of NODAT within 3 years of transplant was $16.2 \%$ overall; $17.7 \%$ with maintenance steroids and $12.3 \%$ without $(P<0.001)$. Patients discharged with steroids had $42 \%$ greater odds of developing NODAT compared with those without steroids (adjusted odds ratio $[\mathrm{AOR}]=1.42,95 \%$ confidence interval $[\mathrm{CI}]=1.27-1.58$, $P<0.001$ ). The maintenance regimen of tacrolimus and mycophenolate mofetil or mycophenolate sodium was associated with $25 \%$ greater odds of developing NODAT $(\mathrm{AOR}=1.25,95 \% \mathrm{CI}=1.08-1.45, P=0.003)$ than the regimen of cyclosporine and mycophenolate mofetil or mycophenolate sodium. Several induction therapies also were associated with lower odds of NODAT compared with no induction. Patients from programs that used steroid-free regimens for a majority of their patients had reduced odds of NODAT compared with patients from programs discharging almost all of their patients on steroid-containing regimens.

Conclusion. The adoption of steroid-free maintenance immunosuppression at discharge from kidney transplantation in selected patients was associated with reduced odds of developing NODAT within 3 years.
\end{abstract}

Keywords: New-onset diabetes after transplant, Steroid-free immunosuppression, Kidney transplantation.

(Transplantation 2011;91: 334-341)

$\mathrm{N}$ ew-onset diabetes after transplant (NODAT) is prevalent in kidney transplant patients. NODAT develops in up to $25 \%$ of previously nondiabetic patients and is associated with an increased risk of posttransplant mortality, aggressive cardiovascular disease, greater health care utilization, and inferior graft survival (1-6). Immunosuppressive medications, including calcineurin inhibitors (CNIs) such as cy-

This work was supported by the Scientific Registry for Transplant Recipients grant 234-2005-37009C, Heath Resources and Services Administration, U.S. Department of Health and Human Services (D.E.S.) and by NIH grant K24 DK062234 (A.O.O.)

A.O.O. received grant support from Pfizer, US, for a separate investigatorinitiated research project and received consulting fees and speaking honoraria from Pfizer, Genzyme, Roche, and Novartis.

Presented at the American Transplant Congress, 2009, Boston, MA.

${ }^{1}$ Division of Nephrology, Department of Internal Medicine, University of Michigan, Ann Arbor, MI.

${ }^{2}$ Scientific Registry of Transplant Recipients, Arbor Research Collaborative for Health, Ann Arbor, MI.

${ }^{3}$ Address correspondence to: Fu Luan, M.D., University of Michigan Health System, 3914 Taubman Center, Box 0364, 1500 East Medical Center Drive, Ann Arbor, MI 48109-0364.

E-mail: fluan@med.umich.edu closporine and tacrolimus, and corticosteroids, are well known risk factors for the development of $\operatorname{NODAT}(7,8)$.

The role of steroids in the development of NODAT has been recognized since the early days of kidney transplantation (9-11). A moderate daily dose of prednisone has been shown to increase insulin resistance, leading to an increased insulin demand to maintain normal glucose tolerance (12). In recent years, many clinical trials have explored the strategy of rapid steroid withdrawal early after kidney transplant and have found survival outcomes comparable with the standard steroid-containing regimens (13-16). In addition, several studies have suggested a potential reduction of the incidence of NODAT when patients were treated with steroid-free maintenance immunosuppression $(14,16,17)$. We explored

\footnotetext{
F.L.L. participated in study design and manuscript preparation; D.E.S. participated in data collection, statistical analyses, and manuscript preparation; and A.O.O. participated in the study design and manuscript preparation.
}

Received 22 April 2010. Revision requested 18 May 2010.

Accepted 26 October 2010.

Copyright (c) 2011 by Lippincott Williams \& Wilkins

ISSN 0041-1337/11/9103-334

DOI: 10.1097/TP.0b013e318203c25f 
the relationship between steroids and NODAT through a retrospective analysis of a national cohort of nondiabetic kidney transplant recipients using the Organ Procurement and Transplantation Network (OPTN)/Scientific Registry of Transplant Recipients data.

\section{RESULTS}

We identified a total of 25,837 adult kidney-only transplant recipients between 2004 and 2006 with no history of diabetes reported to the OPTN before transplantation. Of those 25,837 patients, $6922(26.8 \%)$ were discharged with steroid-free maintenance immunosuppression and 18,915 (73.2\%) with steroids. Table 1 displays characteristics of our sample. Recipient characteristics associated with steroid use at discharge include younger age, African American (AA) race, underlying end-stage renal disease (ESRD) diagnosis of glomerulonephritis or other, longer time on dialysis, higher panel reactive antibodies, repeat transplantation, lower body mass index (BMI), and poorer functional status. An ESRD diagnosis of hypertension or polycystic kidney disease was associated with lower use of steroids at discharge. Donor characteristics associated with greater use of steroids were younger age, male sex, and deceased donor.

Our measure of NODAT was the transplant program's report that the recipient developed diabetes during the follow-up period. A second question asked whether the diabetes required insulin. During the study period, 4194 recipients $(16 \%)$ developed NODAT within the first 3 years after transplantation and $2164(8 \%)$ had NODAT requiring insulin. This is lower than the rates reported in studies using screening fasting-glucose tests or Medicare claims for diabetes to define NODAT, but similar to studies defining diabetes by need for hypoglycemic medication or insulin $(2,6,18)$. Among these 4194 recipients with NODAT, the first report of diabetes for $51 \%$ was at their 6-month follow-up, $20 \%$ at 12 months, $17 \%$ at 24 months, and $12 \%$ at 36 months. The incidence of NODAT was significantly different by use of steroids: $18 \%$ of those discharged on steroids compared with $12 \%$ of those without steroids. The cumulative incidence rates per 1000 person-years, shown in Figure 1, reflect this timing of NODAT, with the highest rate occurring between transplant and 6 months and lower rates as exposure increases with fewer new cases of NODAT.

Table 2 displays the results of a logistic regression model predicting the development of NODAT within the first 3 years after transplant. The odds of developing NODAT were $29 \%$ lower for recipients with steroid-free immunosuppression compared with those with steroids (adjusted odds ratio $[\mathrm{AOR}]=0.71,95 \%$ confidence interval $[\mathrm{CI}]=0.63-0.79$, $P<0.001$ ); conversely, those with steroids had $42 \%$ greater odds of NODAT compared with those without steroids. Recipients with tacrolimus as part of their maintenance regimen had $24 \%$ greater odds of NODAT than those with cyclosporine $(\mathrm{AOR}=1.24,95 \% \mathrm{CI}=1.08-1.42, P=0.002)$, regardless of steroid use (interaction, $P=0.95$ ). Compared with recipients receiving alemtuzumab induction immunosuppression, those receiving antithymocyte globulin had $19 \%$ greater odds of NODAT $(\mathrm{AOR}=1.19,95 \% \mathrm{CI}=1.02-1.39, P=0.03)$, other induction therapies had $27 \%$ greater odds $(\mathrm{AOR}=1.27,95 \%$ $\mathrm{CI}=1.03-1.56, P=0.02$ ), and those with no induction had
$31 \%$ greater odds $(\mathrm{AOR}=1.31,95 \% \mathrm{CI}=1.10-1.55$, $P=0.002)$. Anti-IL-2 receptor antibodies had the same odds as alemtuzumab. Compared with "no induction therapy," the use of anti-IL-2 receptor antibodies was associated with reduced odds of NODAT $(\mathrm{AOR}=0.86,95 \% \mathrm{CI}=0.76-0.98$, $P=0.02)$, as was alemtuzumab $(\mathrm{AOR}=0.77,95 \% \mathrm{CI}=0.65-$ $0.91, P=0.002$ ), while antithymocyte globulin and other therapies were not statistically different. These effects also did not differ by steroid use; however, the interaction of steroids and anti-IL-2 receptor antibodies was close to statistical significance $(P=0.06)$. Anti-IL-2 receptor antibodies were associated with a greater reduction in the odds of NODAT for steroid-free rather than steroid-containing regimens (steroid free: $\mathrm{AOR}=0.61$, $95 \% \mathrm{CI}=0.41-0.90, P=0.01$; steroid containing: $\mathrm{AOR}=0.87$, $95 \% \mathrm{CI}=0.77-0.99, P=0.03)$. Other factors associated with increased odds of NODAT include increased age, non-white race/ ethnicity, repeat transplantation, positive serology for Hepatitis $\mathrm{C}$, higher BMI, and increased time on dialysis. Having hypertension as the cause of ESRD was associated with a higher likelihood of developing NODAT than glomerulonephritis $(\mathrm{AOR}=1.25$, $95 \% \mathrm{CI}=1.15-1.35, P<0.001$ ), as was polycystic kidney disease $(\mathrm{AOR}=1.17,95 \% \mathrm{CI}=1.03-1.32, P=0.01)$. The only significant donor factors increasing risk were having a deceased donor and AA race.

In a separate model, we classified patients according to their maintenance regimens other than steroids and used these regimens instead of choice of CNI, with results shown in Table 3 . The most frequent maintenance regimen was tacrolimus with mycophenolate mofetil (MMF) or mycophenolate sodium (MPA) (tacrolimus-MMF/MPA, 69\%), followed by cyclosporine with MMF/MPA (cyclosporine-MMF/MPA, $11.5 \%$ ), tacrolimus with sirolimus/everolimus (tacrolimussirolimus/everolimus, 4.3\%), tacrolimus alone (tacrolimusonly, $2.7 \%$ ), and cyclosporine with sirolimus/everolimus (cyclosporine-sirolimus/everolimus, 2.4\%). The remaining regimens were grouped as "other" $(10.1 \%)$. Using cyclosporine-MMF/MPA as the reference group, tacrolimus-MMF/ MPA had greater odds of NODAT (AOR $=1.25,95 \%$ $\mathrm{CI}=1.08-1.45, \quad P=0.003) \quad$ as did tacrolimus-sirolimus/ everolimus $(\mathrm{AOR}=1.58,95 \% \mathrm{CI}=1.25-2.00, P<0.001)$.

Because previous research has suggested that AAs are likely to differ from whites in their underlying biological risks for developing diabetes and graft failure (11), we repeated our analysis separately for AA and white race. AAs discharged with steroids had $42 \%$ greater odds of NODAT $(\mathrm{AOR}=1.42$, $95 \% \mathrm{CI}=1.19-1.69, P<0.001)$ than AAs discharged without steroids (23.5\%). The effects of CNIs were not different for AAs and whites, although some regimens were. AAs receiving tacrolimus-sirolimus/everolimus (3.5\%) had 79\% greater odds of NODAT compared with AAs receiving cyclosporine$\mathrm{MMF} / \mathrm{MPA} \quad(\mathrm{AOR}=1.79,95 \% \mathrm{CI}=1.16-2.75, P=0.008)$, while whites receiving tacrolimus-sirolimus/everolimus $(4.4 \%)$ had only $39 \%$ greater odds of NODAT than whites receiving cyclosporine-MMF/MPA ( $\mathrm{AOR}=1.39,95 \%$ $\mathrm{CI}=1.00-1.93, P=0.048)$. The effects of "other" regimens also differed between $\mathrm{AAs}$ and whites (AAs: $\mathrm{AOR}=1.53,95 \%$ $\mathrm{CI}=1.10-2.13, P=0.01$; whites: $\mathrm{AOR}=0.94,95 \% \mathrm{CI}=0.75-$ $1.18, P=0.61)$. The effects of the other factors in the model, including hypertension and BMI, were not statistically different by race, except that AA donor race $(37.6 \%$ of AAs and $5 \%$ of whites) was associated with greater odds of NODAT for 
TABLE 1. Descriptive statistics for selected recipient, donor and transplant characteristics by the inclusion of steroids in the discharge maintenance immunosuppression regimen

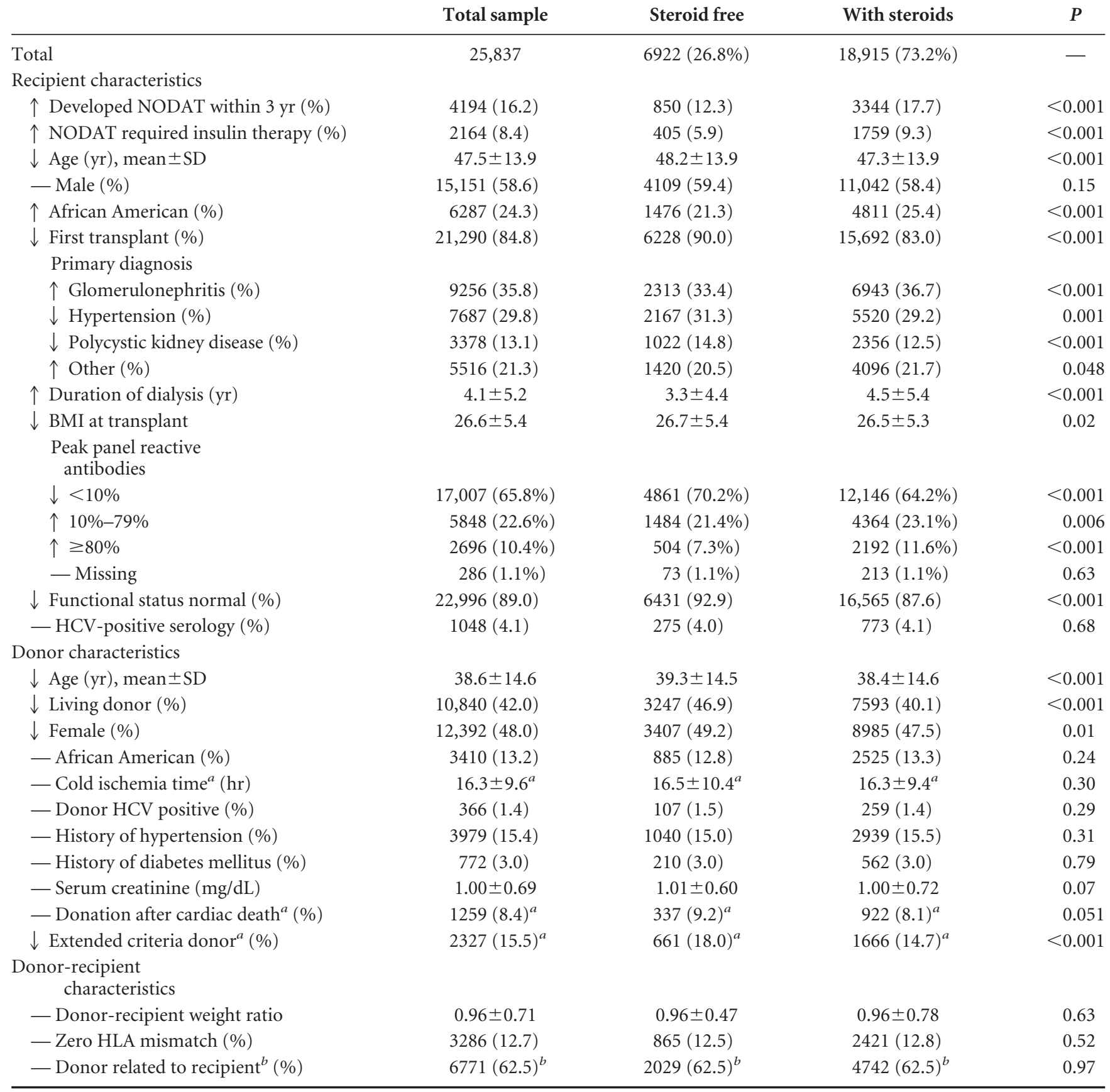

Sample includes adult recipients of kidney-only transplants between 2004 and 2006 discharged alive with a functioning graft and with no indication of diabetes mellitus before transplant.

Arrows indicate direction of bivariate relationship between row characteristic and steroid-free maintenance immunosuppression at discharge: $\uparrow$, associated with greater use of steroids; $\downarrow$, associated with less frequent use of steroids; —, steroid use did not vary by this characteristic.

${ }^{a}$ For deceased donor transplants only $(\mathrm{N}=14,997)$

${ }^{b}$ For living donor transplants only $(\mathrm{N}=10,840)$.

NODAT, new-onset diabetes after transplant; BMI, body mass index; HCV, hepatitis C virus; HLA, human leukocyte antigen (A, B, and DR).

whites than AAs (AAs: $\mathrm{AOR}=1.23,95 \% \mathrm{CI}=1.05-1.43$, $P=0.01$; whites: $\mathrm{AOR}=1.68,95 \% \mathrm{CI}=1.45-1.94, P<0.001$ ).

Because selection bias is likely present when a given patient is chosen to receive steroid-free immunosuppression, we looked at the transplant program's practice pattern re- garding steroids (percentage of recipients discharged with steroids) to reduce this bias. Transplant programs varied in the use of steroid-free immunosuppression, as shown in Figure 2(a). Replacing the individual's own steroid regimen with the program's steroid use and still controlling for person- 


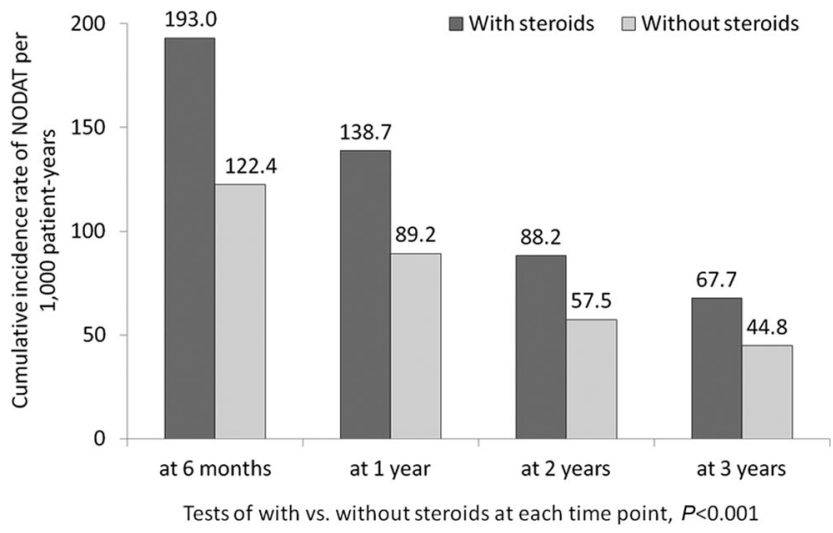

FIGURE 1. The cumulative incidence rates of new-onset diabetes after transplant (NODAT) per 1000 patient years at 6 months and at 1,2 , and 3 years, by use of steroids at discharge.

level factors, we compared recipients from programs that were conservative in the use of steroid-free immunosuppression with those from programs that used it more widely. Figure 2(b) shows odds ratios by program steroid use. Transplantation at a program using steroids for more than $95 \%$ of its patients, compared with a program discharging less than $20 \%$ on steroids, resulted in $48 \%$ greater odds of NODAT. Although this analysis may be less influenced by individual patient characteristics through the use of program-level patterns, it does not eliminate confounding caused when characteristics of patients increase both the need for steroids to prevent rejection and the baseline risk of developing diabetes regardless of transplantation, such as AA race.

\section{DISCUSSION}

Despite the success of kidney transplantation in improving survival compared with dialysis, morbidity and mortality remain elevated among kidney transplant recipients. One reason is the negative effect of immunosuppressive agents on cardiovascular risk factors $(19,20)$, including the development of diabetes. NODAT presents a serious complication incurring significant cardiovascular morbidity and mortality $(4,5)$. The role of steroids in the development of NODAT has been well established and seems dose related ( 9 , 21-23). During the past two decades, investigators have studied steroid withdrawal as a strategy to reduce the incidence of NODAT, with variable success $(10,16,17,24,25)$. However, most of these studies involved relatively small numbers of patients with short follow-up periods, and thus no firm conclusions could be drawn.

The rate of NODAT found in our study is lower than prior nationwide studies $(2,6)$. Both the definition of diabetes and the sample composition of our study differ from these two studies. Our indicator of diabetes is likely more restrictive than the use of two Medicare claims for diabetes and likely misses less severe cases of diabetes that are captured by the Medicare claims. This is reinforced by the finding that $50 \%$ of those who developed NODAT in our study received insulin therapy. By comparison, Woodle et al. (25) found that 21.2\% of their sample developed NODAT by 5 years (two fasting glucose tests $\geq 126 \mathrm{mg} / \mathrm{dL}$ ) but only $7.3 \%$ required insulin (one-third of those with NODAT). Our sample cohort excludes fewer transplant recipients than these studies, which could lead to lower true rates of NODAT. Kasiske et al. (2) limited their sample to first transplants without diabetes and then excluded the $57 \%$ that did not have Medicare as a primary payer, whereas our sample includes higher order transplants and those with other sources of payment. Their sample had a higher prevalence of the risk factors for NODAT identified through their statistical models than the group without Medicare data. Woodward et al. (6) not only used the same source data but also limited their analysis to deceased donor transplants and recipients aged 20 to 65 years, excluding even more recipients compared with our sample.

Additionally, our sample contains more recent transplants, and the strongest risk factors for NODAT have become more prevalent over time, which could lead to higher true rates of NODAT. Recipients in our study (2004-2006) are older and more obese than in Kasiske et al. (1996-2000). The use of tacrolimus has increased dramatically from $24 \%$ in Kasiske et al. to $79 \%$ in our sample, and tacrolimus is known to be more diabetogenic than cyclosporine $(2,3,6,7)$. The use of sirolimus or everolimus has doubled between time frames, from 5\% (1996-2000) to 10\% (2004-2006) $(26,27)$, with some studies suggesting diabetogenic effects of sirolimus $(28,29)$.

However, there have also been immunosuppressionrelated changes that reduce the risk of NODAT, such as the decrease in maintenance steroids s from 98\% in Kasiske et al. to $73 \%$ in our study. Vincenti et al. (16) (in the FREEDOM trial) found similar proportions of patients meeting American Diabetes Association (ADA) criteria for diabetes in the steroid-free and steroid-using groups, but significantly fewer patients required hypoglycemic medication (an indicator more like our measure than the ADA definition) in the steroid-free group. A recent meta-analysis of 30 randomized controlled trials of steroid-sparing immunosuppression regimens suggested reduced risk for NODAT among patients assigned to steroid-sparing regimens (30). Induction therapies, which decreased the odds of NODAT in our study, were used more frequently between 2004 and 2006 (75\%) than between 1996 and 2000 (43\%), and drug-treated acute rejection within the first year after transplant has declined from $25 \%(1996-2000)$ to $10 \%(2004-2006)(26,27)$, lowering overall steroid exposure and possibly lowering the true rate of NODAT. A single-center study comparing recipients from 1995 to 1996 with those from 2004 to 2005 found fewer rejection episodes and a significant decrease in the total dose of methylprednisolone, oral prednisolone, and cyclosporine A in their more recent cohort (31). The fraction with NODAT at 10 weeks, identified by oral glucose tolerance tests, was lower in the newer cohort even though they were older and heavier.

Our study expands on the evidence that the development of NODAT occurs less frequently when patients are discharged with steroid-free maintenance. We included recipients with private insurance and other forms of payment in addition to the half of transplant recipients with Medicare as primary payer. We were also able to compare the variety of immunosuppression strategies used during that time period, including induction therapies and other maintenance drugs in addition to steroids. In our study, tacrolimus use increased the odds of NODAT both with and without concurrent steroid 
TABLE 2. Factors associated with the probability of developing NODAT within 3 years of discharge from the transplant hospitalization $(\mathrm{N}=25,837)$

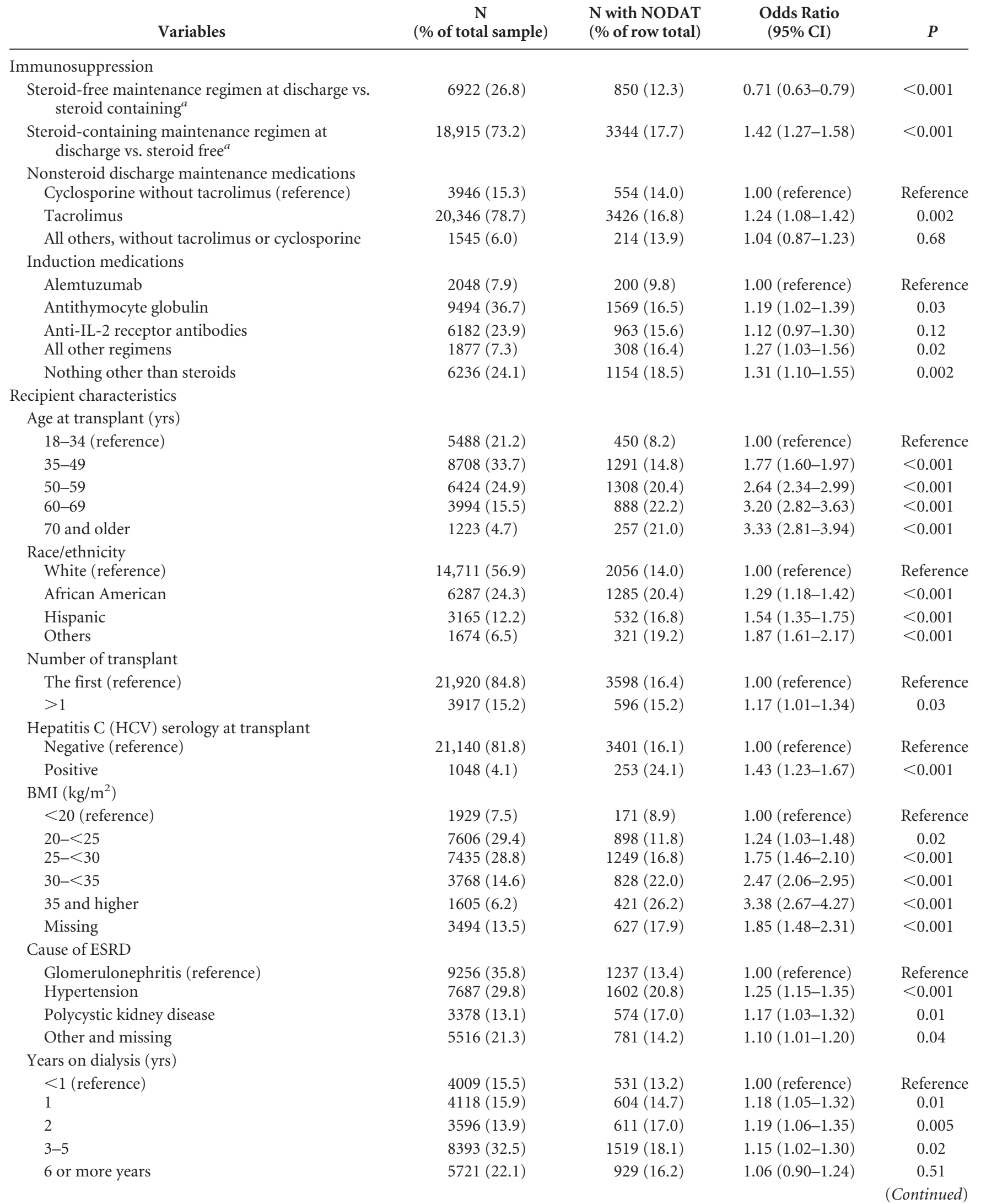


TABLE 2. Continued

\begin{tabular}{|c|c|c|c|c|}
\hline Variables & $\begin{array}{c}\mathrm{N} \\
(\% \text { of total sample) }\end{array}$ & $\begin{array}{l}\text { N with NODAT } \\
\text { (\% of row total) }\end{array}$ & $\begin{array}{l}\text { Odds Ratio } \\
(95 \% \mathrm{CI})\end{array}$ & $P$ \\
\hline \multicolumn{5}{|l|}{ Donor characteristics } \\
\hline Deceased donor & $14,997(58.0)$ & $2712(18.1)$ & $1.15(1.05-1.27)$ & 0.003 \\
\hline Living donor (reference) & $10,840(42.0)$ & $1482(13.7)$ & 1.00 (reference) & Reference \\
\hline \multicolumn{5}{|l|}{ Donor race } \\
\hline African American & $3410(13.2)$ & $729(21.4)$ & $1.32(1.20-1.45)$ & $<0.001$ \\
\hline Hispanic & $3377(13.1)$ & $532(15.8)$ & $0.96(0.85-1.08)$ & 0.46 \\
\hline Asian & $701(2.7)$ & $119(17.0)$ & $1.12(0.90-1.39)$ & 0.30 \\
\hline Other & $208(0.8)$ & $39(18.8)$ & $1.14(0.81-1.62)$ & 0.45 \\
\hline
\end{tabular}

Sample includes adult recipients of kidney-only transplants between 2004 and 2006 discharged alive with a functioning graft and no indication of diabetes mellitus before transplant.

${ }^{a}$ These two rows are equivalent and allow the reader to easily see both the increased odds of NODAT for steroid-containing regimens compared with steroid-free regimens, and the decrease in odds of NODAT for steroid-free regimens compared with steroid-containing regimens.

The model also included the following variables that were not statistically significant at the 0.05 level: recipient sex, recipient HCV status unknown, recipient peak PRA, recipient functional status, recipient private vs. public insurance, donor age, and donor HCV positive. In models not shown, donor sex, cause of death, history of diabetes, history of hypertension, donation after cardiac death, extended criteria donor, donor serum creatinine, machine preservation of kidney, kidney was shared regionally or nationally, living donor is biologically related vs. unrelated, cold ischemia time, and zero HLA mismatch were not significant when included with the characteristics listed in this table.

NODAT, new-onset diabetes after transplant; BMI, body mass index; HCV, hepatitis C virus; ESRD, end-stage renal disease; HLA, human leukocyte antigen (A, B, and DR); PRA, panel reactive antibodies; IL, interleukin.

TABLE 3. Discharge maintenance immunosuppression regimens other than steroids and the probability of developing NODAT within 3 years of discharge

\begin{tabular}{|c|c|c|c|c|}
\hline Maintenance regimens at discharge & $\mathrm{N}$ (\% of total sample) & $\begin{array}{l}\text { N with NODAT } \\
\text { (\% of row total) }\end{array}$ & $\begin{array}{l}\text { Odds ratio } \\
(95 \% \mathrm{CI})\end{array}$ & $P$ \\
\hline Cyclosporine and MMF/MPA (reference) & $2984(11.5)$ & $412(13.8)$ & 1.00 (reference) & Reference \\
\hline Tacrolimus and MMF/MPA & $17,815(69.0)$ & $2968(16.7)$ & $1.25(1.08-1.45)$ & 0.003 \\
\hline Tacrolimus and sirolimus or everolimus & $1105(4.3)$ & $214(19.4)$ & $1.58(1.25-2.00)$ & $<0.001$ \\
\hline Cyclosporine and sirolimus or everolimus & $630(2.4)$ & $92(14.6)$ & $1.34(0.93-1.93)$ & 0.11 \\
\hline Tacrolimus alone & $692(2.7)$ & $114(16.5)$ & $1.19(0.93-1.51)$ & 0.16 \\
\hline
\end{tabular}

Sample includes adult recipients of kidney-only transplants between 2004 and 2006 discharged alive with a functioning graft and no indication of diabetes mellitus before transplant.

This model also included all the variables of Table 2, except for the individual maintenance medications (tacrolimus, cyclosporine, all others). Results for variables not shown were the same as the results in Table 2 .

NODAT, new-onset diabetes after transplant; MMF, mycophenolate mofetil; MPA, mycophenolic acid.

use as did sirolimus/everolimus, whereas several induction therapies lowered them. Our analysis of transplant program-level use of steroids reduces some of the selection bias and supports our individual-level finding that steroid-free immunosuppression is associated with lower odds of NODAT.

As with any registry-based analysis, our study has several limitations. We were constrained by the type of data collected for the registry and its accuracy. No objective measurements of ADA criteria for the definition of diabetes were collected, nor was the date on which the diagnosis was made, which limited the statistical techniques that could be used. The information on immunosuppressive medications in the registry is only collected at discharge and on periodic follow-up reports, rather than every time a change is made, and dosages are not collected. Because different programs often implement early steroid withdrawal on different time- lines soon after discharge, some patients classified as having steroid-containing maintenance in our study may have been steroid free soon after the transplant surgery discharge. In addition, we encountered missing data due to missing reports and the availability of "unknown" as a response option. Most importantly, patients with no maintenance immunosuppressive medications reported at discharge (893 of 31,501 or $2.8 \%)$ and those whose diabetes status at 3 years after transplant were not known (4736 of 31,501 or $15 \%$ ) were excluded, which could have biased our results. Furthermore, patients from the steroid-free and steroid-containing groups differed significantly on several important characteristics that are known to be associated with the development of NODAT. Although the statistical model controlled for these measured variables, these groups possibly differ on important unmeasured variables as well, leading to selection bias. While the 

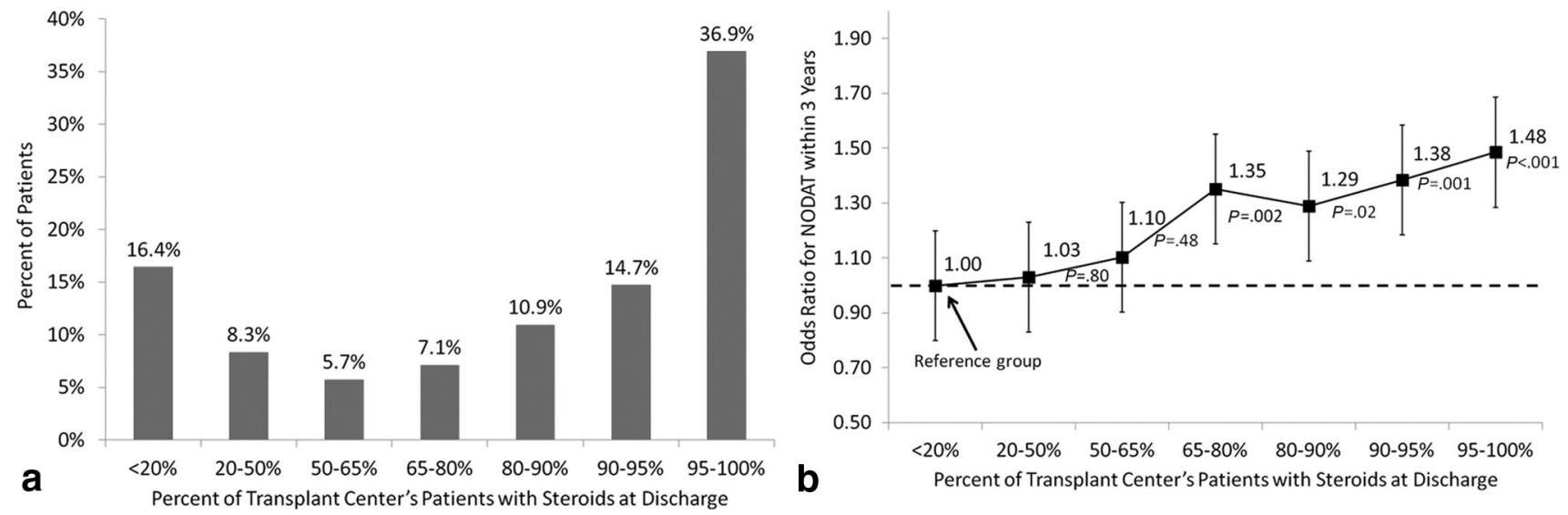

FIGURE 2. The distribution of steroid usage at discharge across US transplant programs (a) and the odds of having new-onset diabetes after transplant (NODAT) for recipients from transplant programs with different levels of steroids use at discharge (b).

program-level analysis likely reduced some of this potential bias, it is likely that the results still suffer from treatment-byindication bias, which could only be eliminated through randomization of the use of steroid-free immunosuppression.

In conclusion, national registry data of kidney transplant recipients selected by their physicians for steroid-free immunosuppression supports the existing evidence that the avoidance of steroids is associated with a significant reduction in the likelihood of developing NODAT and could be considered as one strategy to reduce overall cardiovascular risk in kidney transplant patients.

\section{MATERIALS AND IMETHODS}

\section{Data, Sample, and Dependent Variables}

The Scientific Registry of Transplant Recipients provided data collected by the OPTN from all US kidney transplant programs. The only information on posttransplant diabetes available in the data was one question asking whether the recipient developed diabetes during the follow-up interval, without specifying the criteria to define diabetes, and another one asking whether that diabetes required insulin. The dependent variable was the report of NODAT (with or without insulin) within 3 years of transplant. The sample was all adult, kidney-only transplant recipients engrafted between 2004 and 2006 with no indication of pretransplant diabetes on their waiting list or transplant recipient registrations. Subjects were followed up through February 1, 2010. Those who died or lost graft function before discharge from the transplant hospitalization, had no information on maintenance medications at discharge, or whose 3-year follow-up diabetes status was missing or listed as unknown were excluded from the sample.

\section{Immunosuppressive Regimens}

Patients were classified as being on a steroid-containing or steroid-free regimen according to the report of steroids as a maintenance medication at the time of discharge. Recipients classified as steroid-free may have received steroids during the transplant hospitalization, and steroid use in both groups may have changed after discharge. We also quantified the transplant program's practice pattern regarding steroid maintenance as the fraction of transplant recipients at a program that were discharged with steroids during the calendar year of each transplant in the sample. Using this variable to substitute for the recipient's steroid use at discharge, we examined the differences in the development of NODAT among patients from programs using steroid-free immunosuppression frequently (and for a wider variety of patients) compared with those from programs that rarely use steroid-free regimens (and, hence, selectively).
Induction regimens were classified as rabbit antithymocyte globulin only (Thymoglobulin, Genzyme Corp., Cambridge, MA), anti-IL-2 receptor antibody only (daclizumab [Zenapax, Roche Group, Nutley, NJ] or basiliximab [Simulect, Novartis, Basel, Switzerland]), the anti-CD52 antibody alemtuzumab only (Campath-1H, Genzyme Corp., Cambridge, MA), and all other induction regimens, which included the use of more than one induction agent or other agents such muromonab-CD3 (Orthoclone OKT3, Centocor Ortho Biotech, Horsham, PA), equine antithymocyte globulin (Pfizer, New York, NY), and rituximab (Rituxan, Genentech/Roche Group, San Francisco, CA).

To look specifically at the choice of maintenance CNIs, we classified recipients into three groups: having tacrolimus as part of their regimen, not having tacrolimus but having cyclosporine, or having other drugs that did not include tacrolimus or cyclosporine. In addition, we classified the full regimen of maintenance immunosuppression drugs by examining potential combinations of the following medications: (1) tacrolimus, (2) cyclosporine, (3) mycophenolate mofetil/MMF (Cellcept, Genentech/Roche Group, San Francisco, CA) or mycophenolate sodium/MPA (Myfortic, Novartis, Basel, Switzerland), (4) sirolimus (Rapamune, Wyeth/Pfizer, Madison, NJ) or everolimus (Certican, Norvartis, Basel, Switzerland), and (5) other immunosuppression drugs, such as azathriopine and leflunomide. We identified five specific regimens, shown in Table 3, and grouped the rest as "other maintenance regimens." This set of variables was used in place of the individual CNIs for some models.

\section{Statistical Analysis}

The generalized estimating equations method was used to estimate logistic regression models, allowing recipients to be correlated within transplant programs. Variables used in the analysis, in addition to whether the individual was discharged with steroids, are listed in Tables 2 and 3 . We also performed the same analysis using the programs' use of steroids instead of the recipient's own steroid regimen; all additional covariates remained at the individual-recipient level. All analyses were performed using SAS version 9.2 (SAS Institute, Cary, NC).

\section{REFERENCES}

1. Davidson J, Wilkinson A, Dantal J, et al. New-onset diabetes after transplantation: 2003 International consensus guidelines. Proceedings of an international expert panel meeting. Barcelona, Spain, 19 February 2003. Transplantation 2003; 75(10 suppl): SS3.

2. Kasiske BL, Snyder JJ, Gilbertson D, et al. Diabetes mellitus after kidney transplantation in the United States. Am J Transplant 2003; 3: 178.

3. Heisel O, Heisel R, Balshaw R, et al. New onset diabetes mellitus in patients receiving calcineurin inhibitors: A systematic review and meta-analysis. Am J Transplant 2004; 4: 583. 
4. Hjelmesaeth J, Hartmann A, Leivestad T, et al. The impact of earlydiagnosed new-onset post-transplantation diabetes mellitus on survival and major cardiac events. Kidney Int 2006; 69: 588.

5. Cosio FG, Kudva Y, van der Velde M, et al. New onset hyperglycemia and diabetes are associated with increased cardiovascular risk after kidney transplantation. Kidney Int 2005; 67: 2415.

6. Woodward RS, Schnitzler MA, Baty J, et al. Incidence and cost of new onset diabetes mellitus among U.S. wait-listed and transplanted renal allograft recipients. Am J Transplant 2003; 3: 590.

7. Vincenti F, Friman S, Scheuermann E, et al. Results of an international, randomized trial comparing glucose metabolism disorders and outcome with cyclosporine versus tacrolimus. Am J Transplant 2007; 7: 1506.

8. Rodrigo E, Fernández-Fresnedo G, Valero R, et al. New-onset diabetes after kidney transplantation: Risk factors. J Am Soc Nephrol 2006; 17(12 suppl 3): S291.

9. Friedman EA, Shyh TP, Beyer MM, et al. Posttransplant diabetes in kidney transplant recipients. Am J Nephrol 1985; 5: 196.

10. Hricik DE, Bartucci MR, Moir EJ, et al. Effects of steroid withdrawal on posttransplant diabetes mellitus in cyclosporine-treated renal transplant recipients. Transplantation 1991; 51: 374.

11. Montori VM, Basu A, Erwin PJ, et al. Posttransplantation diabetes: A systematic review of the literature. Diabetes Care 2002; 25: 583.

12. Christiansen E, Andersen HB, Rasmussen K, et al. Pancreatic beta-cell function and glucose metabolism in human segmental pancreas and kidney transplantation. Am J Physiol 1993; 264(3 Pt 1): E441.

13. Matas AJ, Kandaswamy R, Gillingham KJ, et al. Prednisone-free maintenance immunosuppression-a 5-year experience. Am J Transplant 2005; 5: 2473.

14. Kumar MS, Heifets M, Moritz MJ, et al. Safety and efficacy of steroid withdrawal two days after kidney transplantation: Analysis of results at three years. Transplantation 2006; 81: 832.

15. Borrows R, Chan K, Loucaidou M, et al. Five years of steroid sparing in renal transplantation with tacrolimus and mycophenolate mofetil. Transplantation 2006; 81: 125.

16. Vincenti F, Schena FP, Paraskevas S, et al. A randomized, multicenter study of steroid avoidance, early steroid withdrawal or standard steroid therapy in kidney transplant recipients. Am J Transplant 2008; 8: 307.

17. Rostaing L, Cantarovich D, Mourad G, et al. Corticosteroid-free immunosuppression with tacrolimus, mycophenolate mofetil, and daclizumab induction in renal transplantation. Transplantation 2005; 79: 807.

18. Matas AJ, Gillingham KJ, Humar A, et al. Posttransplant diabetes mellitus and acute rejection: Impact on kidney transplant outcome. Am J Transplant 2008; 85: 338 .
19. Curtis JJ. Cyclosporine and posttransplant hypertension. J Am Soc Nephrol 1992; 2(12 suppl): S243.

20. Hjelmesaeth J, Hartmann A, Midtvedt K, et al. Metabolic cardiovascular syndrome after renal transplantation. Nephrol Dial Transplant 2001; 16: 1047.

21. Arner P, Gunnarsson R, Blomdahl S, et al. Some characteristics of steroid diabetes: A study in renal-transplant recipients receiving high-dose corticosteroid therapy. Diabetes Care 1983; 6: 23.

22. Hjelmesaeth J, Hartmann A, Kofstad J, et al. Tapering off prednisolone and cyclosporin the first year after renal transplantation: The effect on glucose tolerance. Nephrol Dial Transplant 2001; 16: 829.

23. Weir MR, Fink JC. Risk for posttransplant diabetes mellitus with current immunosuppressive medications. Am J Kidney Dis 1999; 34: 1 .

24. Hollander AA, Hene RJ, Hermans J, et al. Late prednisone withdrawal in cyclosporine-treated kidney transplant patients: A randomized study. J Am Soc Nephrol 1997; 8: 294.

25. Woodle ES, First MR, Pirsch J, et al. A prospective, randomized, double-blind, placebo-controlled multicenter trial comparing early (7 day) corticosteroid cessation versus long-term, low-dose corticosteroid therapy. Ann Surg 2008; 248: 564.

26. 2008 Annual Report of the U.S. Organ Procurement and Transplantation Network and the Scientific Registry of Transplant Recipients: Transplant Data 1998-2007. U.S. Department of Health and Human Services, Health Resources and Services Administration, Healthcare Systems Bureau, Division of Transplantation, Rockville, MD.

27. 2006 Annual Report of the U.S. Organ Procurement and Transplantation Network and the Scientific Registry of Transplant Recipients: Transplant Data 1996-2005. U.S. Department of Health and Human Services, Health Resources and Services Administration, Healthcare Systems Bureau, Division of Transplantation, Rockville, MD.

28. Romagnoli J, Citterio F, Nanni G, et al. Incidence of posttransplant diabetes mellitus in kidney transplant recipients immunosuppressed with sirolimus in combination with cyclosporine. Transplant Proc 2006; 38: 1034.

29. Johnston O, Rose CL, Webster AC, et al. Sirolimus is associated with new-onset diabetes in kidney transplant recipients. J Am Soc Nephrol 2008; 19: 1411.

30. Pascual J, Zamora J, Galeano C, et al. Steroid avoidance or withdrawal for kidney transplant recipients. Cochrane Database Syst Rev 2009; (1): CD005632.

31. Valderhaug TG, Hjelmesœth J, Rollag H, et al. Reduced incidence of new-onset posttransplantation diabetes mellitus during the last decade. Transplantation 2007; 84: 1125. 